\title{
Hemolytic uremic syndrome - a case presentation
}

\author{
Teodora Adam¹, Cornel Olimpiu Aldea', Sorin Claudiu Man² \\ ${ }^{1}$ Clinical Emergency Hospital for Children, Cluj-Napoca, Romania \\ ${ }^{2}$ Discipline Pediatrics III, University of Medicine and Pharmacy "Iuliu Hatieganu", Cluj-Napoca, Romania
}

\begin{abstract}
The presentation aims to illustrate in a didactic manner the clinical aspects, laboratory findings and the diagnostic steps in a case of hemolytic uremic syndrome (HUS), also pointing out the importance of differential diagnosis for the triad consisting of microangiopathic hemolytic anemia, thrombocytopenia and acute kidney injury. The clinician's approach regarding the prognostic factors is also depicted, alongside the hallmarks concerning the treatment of HUS, the aspects of acute kidney injury monitoring and the need for a long term follow-up of HUS patients.
\end{abstract}

Keywords: hemolytic uremic syndrome, microangiopathic hemolytic anemia, thrombocytopenia, acute kidney injury, child

\section{INTRODUCTION}

In day to day practice, pediatric physicians are relying on the history and physical examination in order to formulate several hypotheses and establish a clinical diagnosis, also taking into account the most frequently encountered causes and excluding of life-threatening conditions. The present case aims to point out the importance of such a diagnostic approach.

\section{HISTORY}

A two and a half-year-old female patient with a history of acute respiratory tract infections was transferred to our hospital for pallor and oliguria.

The disease onset was a week prior with complaints of fever, rhinorrhea and cough, treated with acetaminophen and trimethoprim-sulfamethoxazole. The patient associated watery diarrhea and vomiting, dehydration, pallor and an altered general appearance, symptoms that prompted the initial hospitalization. On admission, the patient presented elevated blood pressure $(130 / 80 \mathrm{mmHg})$, spasticity and drowsiness with periods of irritability, oliguria and macroscopic hematuria. Hemoglobin $(6.5 \mathrm{~g} / \mathrm{dl})$ and thrombocyte $(30,000 / \mu \mathrm{l})$ levels were low, with elevated levels of urea $(80 \mathrm{mg} /$ dl) and creatinine $(3.4 \mathrm{mg} / \mathrm{dl})$. The patient was treated with furosemide and mannitol, but presented generalized seizures controlled with diazepam and phenobarbital.

\section{CLINICAL EXAM}

Upon transfer in our clinic, BP was 100/60 $\mathrm{mmHg}\left(95-99^{\text {th }}\right.$ percentile $/<90^{\text {th }}$ percentile), with no urine output in the last 12 hours, pallor, altered mental status, drowsiness with difficulty responding to verbal stimuli. 


\section{CLINICAL DIAGNOSIS}

Acute gastroenteritis followed by oliguria and pallor is suggesting in general hemolytic uremic syndrome (HUS).

\section{LABORATORY FINDINGS}

Initial laboratory tests were directed to confirm the presence of diagnostic criteria for HUS: (1) microangiopathic hemolytic anemia, (2) thrombocytopenia and (3) acute kidney injury (AKI) (for the last one the diagnostic criteria are: (a) azotemia, (b) electrolyte disturbances, and (c) acid-base balance disturbances) (1-4).

Blood tests showed microangiopathic (schistocytes on peripheral blood smear) hemolytic (reticulocyte count 6\%) anemia (hemoglobin $7 \mathrm{~g} / \mathrm{dl}$ ) and

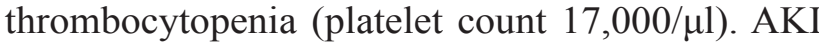
was confirmed by the presence of (a) azotemia (urea $200 \mathrm{mg} / \mathrm{dl}$ and creatinine $4.8 \mathrm{mg} / \mathrm{dl}$ ), (b) electrolyte disturbances (sodium $120 \mathrm{mmol} / \mathrm{l}$, ionized calcium $1.03 \mathrm{mmol} / \mathrm{l}$ ) and (c) metabolic acidosis (pH 7.31, bicarbonate $14.8 \mathrm{mmol} / \mathrm{l})$. Interestingly, the case presented potassium at lower limit (3.7 $\mathrm{mmol} / \mathrm{l}$ ), whereas the typical disturbance in AKI is hyperkalemia. This finding could be explained by the prodrome of diarrhea and vomiting which both contributed to potassium deficiency.

The signs and symptoms (pallor, oliguria, onset after an acute gastroenteritis) raised the suspicion of HUS, which was supported by laboratory tests showing microangiopathic hemolytic anemia, thrombocytopenia and AKI with acidosis and electrolyte imbalance.

So, the laboratory tests confirmed the presence of the three criteria for the diagnosis of HUS. Considering this, is it necessary to perform a differential diagnosis? The answer is ,yes“, due to the fact that the combination of microangiopathic hemolytic anemia, thrombocytopenia and AKI can be found in several other diseases.

\section{DIFFERENTIAL DIAGNOSIS}

Differential diagnosis should be performed to exclude other diseases that present with microangiopathic hemolytic anemia, thrombocytopenia and AKI (Table 1) (1-3).

In general, final diagnosis should:

1) localize the pathology ("anatomical" diagnosis) $\rightarrow$ in this case HUS;

2) pinpoint the causative factor (etiological diagnosis) $\rightarrow$ in this case unidentified (the stool culture was negative due to previous antibiotic therapy);

3) describe how the disease impaired organ function (functional diagnosis) $\rightarrow$ AKI;

4) characterize the clinical type of the disease (clinical type diagnosis) $\rightarrow$ HUS with diarrheal prodrome;

5) identify the complications associated with the clinical course or treatment (diagnosis of complications) $\rightarrow$ Urinary tract infection (UTI) associated with bladder catheterization (urine culture was positive with Escherichia coli 100,000 UFC/ $\mathrm{ml})$.

Therefore, the final diagnosis for the present case was HUS with diarrheal prodrome caused by unidentified microorganism complicated with UTI.

\section{PROGNOSIS}

Elements that predict an immediate negative outcome in HUS are:

- Anuria $>7$ days;

- Oliguria $>7$ days;

TABLE 1. Differential diagnosis of HUS

\begin{tabular}{|c|c|}
\hline Disease considered for differential diagnosis & Elements that do not support the diagnosis \\
\hline Systemic lupus erythematosus & $\begin{array}{ll}\text { - } & \text { Age }<10 \text { years } \\
\text { - } & \text { No rash } \\
\text { - } & \text { No arthritis } \\
& \text { Normal level of } \mathrm{C3}(72 \mathrm{mg} / \mathrm{dl}) \\
\end{array}$ \\
\hline Malignant hypertension & - $\quad$ BP $110 / 60 \mathrm{mmHg}\left(95-99^{\text {th }}\right.$ percentile $/<90^{\text {th }}$ percentile $)$ \\
\hline Bilateral renal veins thrombosis & - Normal kidney size by ultrasound (usually markedly increased in thrombosis) \\
\hline Disseminated intravascular coagulation & $\begin{array}{l}\text { - } \quad \text { Trigger factor is usually sepsis } \\
\text { - } \quad \text { Normal prothrombin time (11.5 s) } \\
\text { - } \quad \text { Normal activated partial thromboplastin time (22 s) }\end{array}$ \\
\hline Thrombotic thrombocytopenic purpura & $\begin{array}{l}\text { - } \\
\text { - }\end{array}$ \\
\hline
\end{tabular}


- Age $<1$ year old or $>5$ years old;

- Absence of diarrhea;

- Severe hypertension;

- Central nervous changes;

- Leukocytes $>20,000 / \mu \mathrm{l}$;

- Hypocalcemia (5).

The patient presented two of these poor prognostic features (central nervous changes, hypocalcemia), and the white blood count was $17,000 / \mu 1$.

\section{Treatment plan included:}

- treatment of anemia with packed red blood cells: $2 \times 15 \mathrm{ml} / \mathrm{kg}$;

- thrombocytopenia management with aspirin therapy: $8 \mathrm{mg} / \mathrm{kg} / \mathrm{day}$;

- therapy of AKI with intravenous furosemide $2 \mathrm{mg} / \mathrm{kg} /$ day; furosemide does not cure the $\mathrm{AKI}$, but it can convert an oliguric AKI to a non-oliguric AKI, which was the case;

- correction of electrolyte imbalance;

- treatment of complication: antibiotic therapy for UTI with a corrected dose accordingly to creatinine clearance value (ceftazidime 20 $\mathrm{mg} / \mathrm{kg} /$ day for a creatinine clearance of 15 to $6 \mathrm{ml} / \mathrm{min} / 1.73 \mathrm{~m}^{2}$ ).

\section{Evolution and follow-up}

In order to understand the clinical course of an AKI, one should know several facts:

- In AKI the glomerular filtration rate (GFR) ranges between 0 to $10 \%$ of normal;

- The decrease of GFR value occurs in the first 24 hours after the kidney injury;

- Creatinine level increases slowly (about 1 $\mathrm{mg} / \mathrm{dl} /$ day) and does not follow the abrupt decrease in GFR (4,6-8).

There are two extreme possibilities: either GFR drops to zero (Fig. 1) or GFR drops to $10 \%$ of normal (Fig. 2). If GFR dropps to zero (Fig. 1), the creatinine level would continuously increase until dialysis is started or kidney function is restored (4,6-8).

If GFR dropps to $10 \%$ of normal (Fig. 2), the creatinine would slowly increase until it reaches a plateau that will accurately reflect GFR. In the present case, the creatinine value of $3.4 \mathrm{mg} / \mathrm{dL}$ did not reflect the actual GFR; instead, the plateau value of $4.8 \mathrm{mg} / \mathrm{dl}$ showed the true severity of the kidney function impairment. Therefore, in the present case, an increase of the creatinine level from 3.4 to 4.8 $\mathrm{mg} / \mathrm{dl}$ did not signify an augmentation in disease

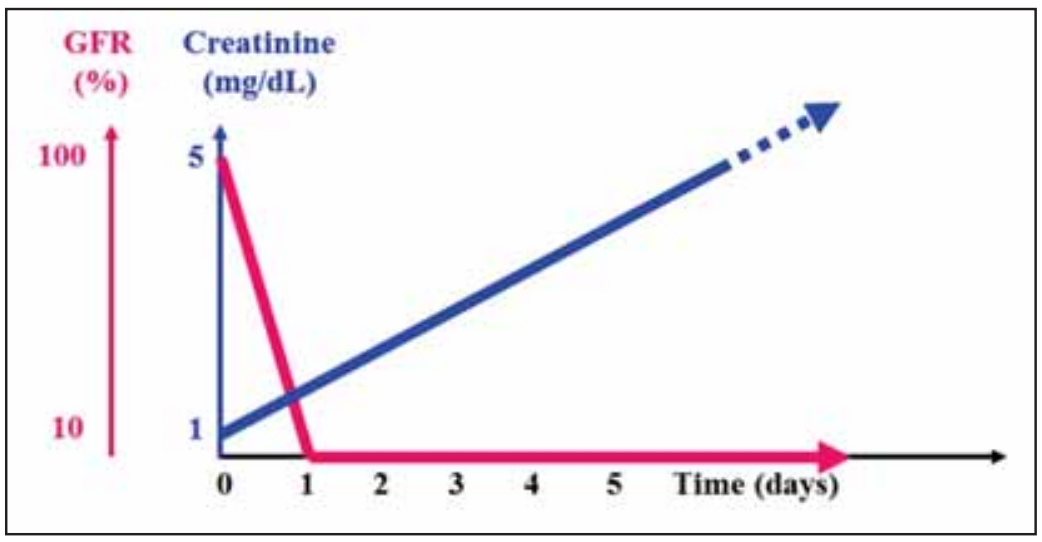

FIGURE 1. Course of serum creatinine if GFR drops to zero

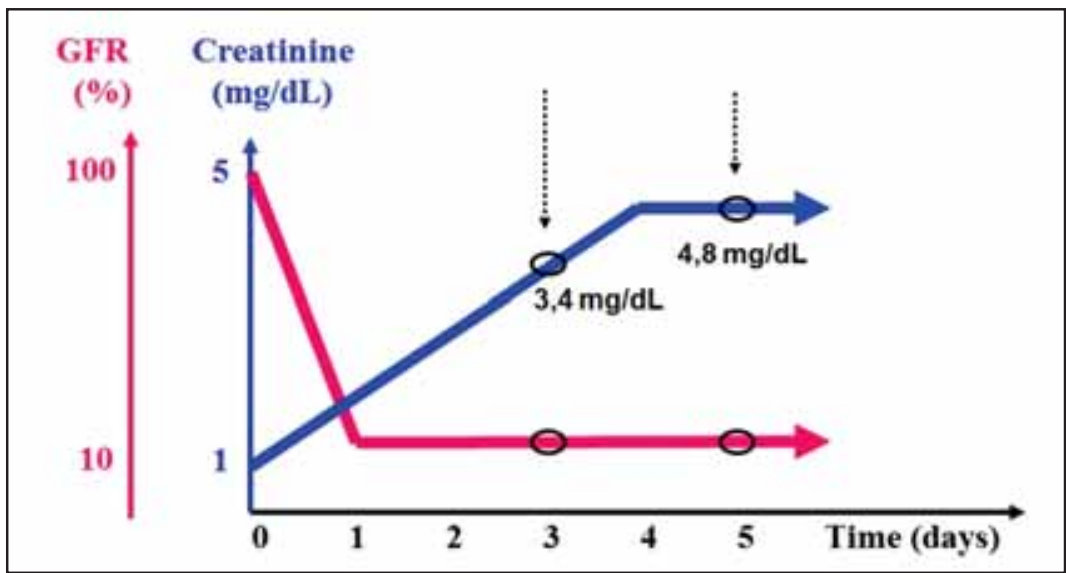

FIGURE 2. Course of serum creatine if GFR drops to $10 \%$ of normal value 


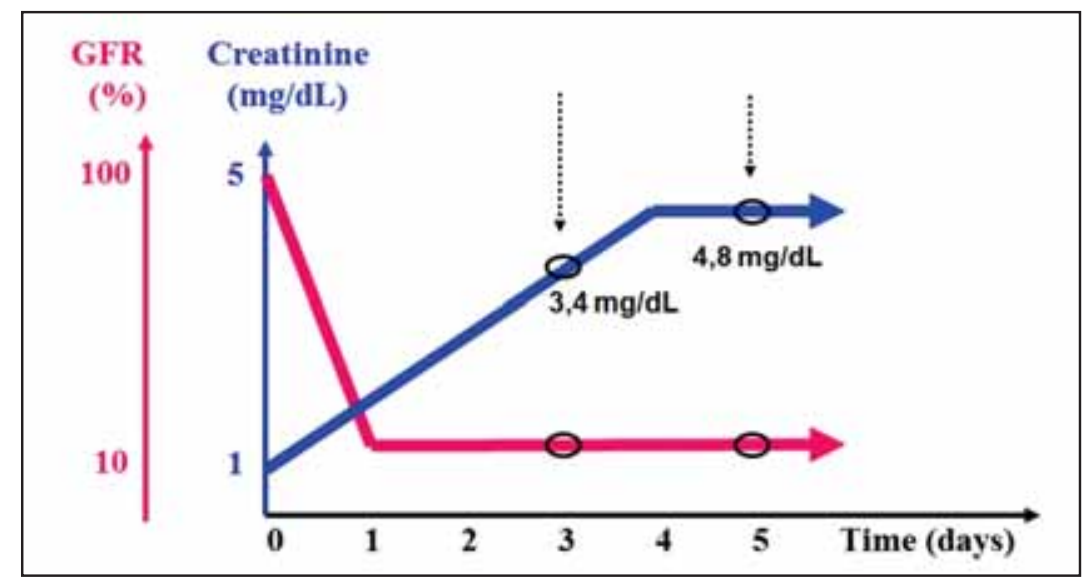

FIGURE 3. Course of serum creatinine and thrombocyte count after giving aspirin

severity, simply the $3.4 \mathrm{mg} / \mathrm{dl}$ value was not yet reflective of the real GFR. There are at least two practical points derived from this: 1 ) the creatinine levels should be measured daily at least until a plateau is reached, and 2) for drug dose calculations in AKI one should assume that GFR is less than $10 \%$, irrespective of the creatinine level at a given measurement (bearing the fact that in the first days of the disease creatinine level might not accurately reflect the impairment in kidney function) $(4,6-8)$.

Aspirin treatment was initiated in order to control thrombocytopenia with a favorable evolution

\section{REFERENCES}

1. Brocklebank V., Wood K.M., Kavanagh D. Thrombotic Microangiopathy and the Kidney. Clin J Am Soc Nephrol 2017; [Epub ahead of print].

2. Laurence J., Haller H., Mannucci P.M., Nangaku M., Praga M., Rodriguez de Cordoba S. Atypical hemolytic uremic syndrome (aHUS): essential aspects of an accurate diagnosis. Clin Adv Hematol Oncol 2016;14 Suppl 11:2-15.

3. Shen Y.M. Clinical evaluation of thrombotic microangiopathy: identification of patients with suspected atypical hemolytic uremic syndrome. Thromb J 2016;14:19.

4. Ostermann M., Joannidis M. Acute kidney injury 2016: diagnosis and diagnostic workup. Crit Care 2016; 20:299. resulting in correction of thrombocyte count as shown in Fig. 3.

At discharge, the child presented mild anemia (hemoglobin $10.5 \mathrm{~g} / \mathrm{dl}$ ), normal renal function (urea $29 \mathrm{mg} / \mathrm{dl}$, creatinine $0.78 \mathrm{mg} / \mathrm{dl}$ ), normal thrombocyte count and a normal urine exam (3-4 red blood cell/high-power field, albumin 1+).

The follow-up of the case until 18 years of age did not identify any long-term effects (hypertension, proteinuria, hematuria, chronic kidney disease) of HUS.

Conflict of interest: none declared Financial support: none declared

5. Oakes R.S., Siegler R.L., McReynolds M.A., Pysher T., Pavia A.T. Predictors of fatality in postdiarrheal hemolytic uremic syndrome. Pediatrics 2006; 117:1656-62.

6. Fortenberry J.D., Paden M.L., Goldstein S.L. Acute kidney injury in children: an update on diagnosis and treatment. Pediatric clinics of North America 2013; 60:669-88.

7. Kher K.K., Schnaper H.W., Greenbaum L.A. Clinical pediatric nephrology. Third edition. ed. Boca Raton: CRC Press; 2016.

8. Geary D.F. Pediatric kidney disease. 2nd edition. ed. New York, NY: Springer Berlin Heidelberg; 2016. 